\title{
New insights on key genes involved in drought stress response of barley: gene networks reconstruction, hub, and promoter analysis
}

\author{
Seyedeh Mehri Javadi ${ }^{1}$, Zahra-Sadat Shobbar ${ }^{2 *}$, Asa Ebrahimi ${ }^{1}$ and Maryam Shahbazi ${ }^{3}$
}

\begin{abstract}
Background: Barley (Hordeum vulgare L.) is one of the most important cereals worldwide. Although this crop is drought-tolerant, water deficiency negatively affects its growth and production. To detect key genes involved in drought tolerance in barley, a reconstruction of the related gene network and discovery of the hub genes would help. Here, drought-responsive genes in barley were collected through analysis of the available microarray datasets ( $-5 \geq$ Fold change $\geq 5$, adjusted $p$ value $\leq 0.05$ ). Protein-protein interaction (PPI) networks were reconstructed.

Results: The hub genes were identified by Cytoscape software using three Cyto-hubba algorithms (Degree, Closeness, and MNC), leading to the identification of 17 and 16 non-redundant genes at vegetative and reproductive stages, respectively. These genes consist of some transcription factors such as HvVp1, HvERF4, HvFUS3, HvCBF6, DRF1.3, HvNAC6, HvCO5, and HvWRKY42, which belong to AP2, NAC, Zinc-finger, and WRKY families. In addition, the expression pattern of four hub genes was compared between the two studied cultivars, i.e., "Yousef" (drought-tolerant) and "Morocco" (susceptible). The results of real-time PCR revealed that the expression patterns corresponded well with those determined by the microarray. Also, promoter analysis revealed that some TF families, including AP2, NAC, Trihelix, MYB, and one modular (composed of two HD-ZIP TFs), had a binding site in 85\% of promoters of the drought-responsive genes and of the hub genes in barley.

Conclusions: The identified hub genes, especially those from AP2 and NAC families, might be among key TFs that regulate drought-stress response in barley and are suggested as promising candidate genes for further functional analysis.
\end{abstract}

Keywords: Barley, Drought stress, Biological networks, Hub gene, Promoter analysis

\section{Background}

Barley (Hordeum vulgare L.) is one of the most important cereals in the world [1]. The plant is ecologically adaptable to a wide range of environments and generally has higher drought tolerance compared to other cereals. Barley is usually regarded as a suitable model for studying abiotic stress because of high genomic diversity and

\footnotetext{
* Correspondence: Shobbar@abrii.ac.ir

${ }^{2}$ Department of Systems Biology, Agricultural Biotechnology Research Institute of Iran (ABRII), Agricultural Research, Education and Extension Organization (AREEO), Karaj, Iran

Full list of author information is available at the end of the article
}

unique physiological/morphological properties [2]. Drought stress is among the strongest environmentally effective parameters limiting barley's growth and productivity $[3,4]$. Since climate change has reduced annual amounts of rainfall and increased the temperature in most areas [5-7], drought tolerance is considered as a complex character that includes a series of physiological, morphological, and biochemical changes in the plant [8]. These can include earliness, reduced leaf area, leaf sprain, increased efficiency of the root system, reduced tillering, and accretion of osmoprotectants [9]. Extensive 
studies have been conducted to identify the genes involved in each of these responses in barley [10-17].

To better understand drought tolerance and its mechanisms, the identification of DEGs in stress conditions could be a preliminary step, while reconstructing the related network and detecting the key genes would be the next necessary steps. By completing many genome projects, researchers have turned their attention from investigating individual genes to studying their interaction networks, since the components do not operate independently inside the cell, and their performance and features are defined in operation with other elements. The discovery and analysis of cell biological processes is one of the main objectives in the post-genomic period [18]. Cellular processes are adjusted using the interaction between different molecules such as protein, DNA ,and metabolites $[19,20]$. Regarding the crucial role of regulatory elements in plant responses to drought stress [21], promoter analysis is a powerful method to understand the mechanism [21]. Currently, many studies indicate that the barley transcription factor is involved in drought tolerance. Sazegari et al. [22] reported that ERF/AP2, C2C2-Dof, and bHLH transcription factor families are regulatory components of the transcriptional cascade involved in priminginduced tolerance. The expression of the gene for the barley MYB transcription factor, $H v M Y B 1$, was reportedly upregulated in roots and leaves by drought, which reduced stomatal conductance, enhanced proline content, and reduced ROS levels and catalase activity [23]. Ju et al. [24] reported that under drought conditions the $V v N A C 17$-overexpression lines had lower malondialdehyde and $\mathrm{H}_{2} \mathrm{O}_{2}$ contents, but higher peroxidase, superoxide dismutase, catalase activity, and proline content. The WRKY belongs to an important plantspecific transcription factor family which is involved in response to environmental stresses. Analyzing the available microarray data revealed eight candidate WRKY genes which were upregulated under drought and salinity stresses compared to the optimum conditions at seedling stage in barley [25]. In the current research, we attempted to collect highly drought-responsive genes in barley through analysis of all the available related microarray data [10-14]. Then, the gene regulatory and protein-protein interaction (PPI) networks were reconstructed using the resultant gene list. To find the key genes regulating the drought stress response, a hub analysis was performed. Moreover, promoter analysis revealed the most common transcription factor families that have binding sites in the promoters of the highly droughtresponsive genes. Finally, real-time PCR was used for validating some of the most promising candidate genes.

\section{Methods}

Identification of drought-inducible genes in barley

All the available microarray data (up to the time of the study) on barley (Hordeum vulgare) regarding its responses to drought stress (GSE3170, GSE6990,
GSE15970, and GSE17669 Datasets) were downloaded at https://www.ncbi.nlm.nih.gov/geo/ (Table 1). Data normalization was done using the RMA algorithm by Expression console software, Version 1.3.1 (https://www. thermofisher.com/.../affymetrix-expression-consolesoftware.html). Differentially expressed genes with a fold change of $\geq 5$ and $\leq-5$ and adjusted $p$ value $\leq 0.05$ were identified using FlexArray software (ver1.6.3) (http://www.affymetrix.com/products/software/ compatible/index.affx). Redundant genes that were obtained from different microarray datasets were removed. The ortholog of these non-redundant genes in Arabidopsis (Arabidopsis thaliana) was identified using BLASTx (https://blast.ncbi.nlm.nih.gov/Blast.cgi) and BLASTn [28]. These orthologous genes in Arabidopsis were used for network reconstruction.

\section{Reconstruction of genes and protein-protein interactions (PPIs) networks and the hub analysis}

The mentioned list of genes was analyzed in the webbased application of STRING ver.10 [29] (http://string$\mathrm{db} . o r g)$, and the protein-protein interactions list was prepared. Cytoscape (Ver 3.4) and plugin of Cyto-Hubba were used for drawing the protein-protein interaction network and for identifying highly connected protein nodes (hubs) [30]. Three computational algorithms of Cyto-Hubba named Degree, Closeness, and MNC were used for detecting hub genes (10 nodes with the most interactions).

\section{Gene Ontology enrichment analysis of DEGs}

Gene Ontology (GO) enrichment was analyzed at http:// bioinfo.cau.edu.cn/agriGO. Annotations were made for GO terms based on biological processes (BP), cellular components (CC), and molecular function (MF) [31]. The significance of the $\mathrm{GO}$ terms was tested through the Fisher's exact test $(P<0.05)$ [32].

\section{Promoter analysis}

Promoter sequences of the genes (identified orthologs in Arabidopsis) were derived using the Gene2Promoter program available in Genomatix Software Suite (https:// www.genomatix.de). The promoter areas of the genes were defined as 1000 and $200 \mathrm{bp}$ (upstream and downstream, respectively) of the transcription initiation site in the intended gene. Common TFs and framework analyses were used respectively for detecting the common transcription factors and common modules, which were present in $85 \%$ of the promoters. The similarity level of the core was considered to be 1 in the conducted analysis, and the level of similarity matrix was set on the optimized option. 
Table 1 The microarray data series used in this study

\begin{tabular}{llllll}
\hline Series & Technology & Genotype & Tissue & Developmental Stage & Reference \\
\hline GSE3170 & $\begin{array}{l}\text { GeneChip arrays- } \\
\text { Affymetrix }\end{array}$ & $\begin{array}{l}\text { Barke, Morex and } \\
\text { Stepoe }\end{array}$ & Seedlings & Vegetative stage/seedlings & Cui et al. [26] \\
GSE6990 & $\begin{array}{l}\text { GeneChip arrays- } \\
\text { Affymetrix }\end{array}$ & Morex & Crown & Vegetative stage/seedlings & Tommasini et al. \\
GSE15970 & $\begin{array}{l}\text { GeneChip arrays- } \\
\text { Affymetrix }\end{array}$ & Martin, HS41-1, & Flag leaves & Reproductive stage/post & Guo et al. [12] \\
GSE17669 & $\begin{array}{l}\text { GeneChip arrays- } \\
\text { Affymetrix }\end{array}$ & Morex & Spikes (Lemmas, paleas, awns, and & Reproductive stage/grain- & Abebe et al. [13] \\
& & seeds) & filling & & \\
\hline
\end{tabular}

\section{Plant material and experimental treatment}

The seeds of two spring barley genotypes (i.e., "Morocco" as a drought-susceptible and "Yousef" as a drought-tolerant variety of barley) were used in experiments laid out as RCBD for the two treatments (wellwatered and drought-stressed) and three replicates in a greenhouse. The plants were cultivated in well-watered settings until flowering, and the drought treatment begun at this time by withholding irrigation. Samplings were performed randomly among the main stems with the same height, i.e., 21 days after anthesis (DAA). The samples were subject to rapid freezing in liquid nitrogen and were kept at $-80{ }^{\circ} \mathrm{C}$ for the experiments.

\section{Real-time PCR}

To validate the bioinformatic data, four hub genes (HvDRFI.3, HvCBF6, HvCO5, and HvWRKY42) were selected. Total RNA was extracted from penultimate tissues of the barley genotypes in three biological replicates using Trizol reagent as instructed by the producer. To confirm the removal of genomic DNA, RNA samples were subjected to RNase-free DNase treatment (Promega, USA). cDNA was synthesized by the iScript kit. The qRT-PCR was carried out by a LightCycler 96 Real-time PCR System and iQ Syber Green Supermix kit as per the company's instructions. The gene-specific primers (Supplementary Table 9) were designed using Oligo 7.0. The qRT-PCR was implemented for three replications of both control and drought-treated samples from both barley genotypes with a LightCycler 96 RealTime PCR System and SYBR Premix EX Taq II as described in the producer's guidelines. Relative mRNA abundance (in fold change) was measured via the deltadelta $\mathrm{Ct}(\Delta \Delta \mathrm{Ct})$ technique [33] after normalization of the $\mathrm{Ct}$ value for individual genes versus Actin (AY145451.1) as the endogenic control. Fold change was estimated using the REST software to analyze qPCR data (based on the Pfaffl method) [34].

\section{Results}

Identification of drought-responsive genes in barley A total of 250 differentially expressed genes (DEGs) (5 $\leq$ fold change $\leq-5$ ) were obtained, while 134 and 116 of which were upregulated and downregulated, respectively, in drought conditions based on the microarray data analysis (Table 1) after removal of the duplicated genes (Supplementary Tables 1 and 2). We further classified the DEGs into vegetative and reproductive organs including 215 and 35 genes, respectively.

\section{Reconstruction of genes and PPIs networks, in addition to the hub analysis}

The gene and PPI networks were reconstructed (Fig. 1 and Supplementary Fig. 1). The hub analysis led to the identification of 17 and 16 non-redundant genes with the most interactions being in vegetative and reproductive organs, respectively (Supplementary Tables 3 and 4). A significant proportion of the hub genes was related to regulatory processes such as transcription and expression regulation (e.g., $H \nu W R K Y 42, H \nu V P 1, H \nu C B F 6$, and $D R F 1.3)$, which belong to transcription factor families.

\section{Gene Ontology enrichment analysis of the DEGs}

The SEA was utilized for testing the GO enrichment according to the 250 DEGs in vegetative and reproductive organs and hub genes. Several items were detected as predominant terms, i.e., metabolic processes, stress responses, responses to hormonal stimuli, hormonefacilitated signaling pathways, transportation, protein metabolic processes, gene expression, transcription, and regularization of $\mathrm{BP}$. The most significant MF terms were binding, nucleotide-binding, and transcription activator activity. Concerning the $\mathrm{CC}$ ontology, the enriched term was the nucleus (Figs. 2, 3, and 4).

\section{Promoter analysis}

Based on the results, some transcription factor families including AP2, bZIP, HD-ZIP, MYB, NAC, MADS, Trihelix, and WRKY had binding sites in more than $85 \%(P$ value $<0.05$ ) of the drought-responsive genes (Fig. 5 and supplementary Table 5) and the hub genes (Fig. 6 and supplementary Table 6). Interestingly, the number of binding sites in these transcription factors were predicted and significantly high in the promoters of the differentially expressed genes and in the hub genes (Figs. 5 and 6 and supplementary Tables 5 and 6). For instance, 


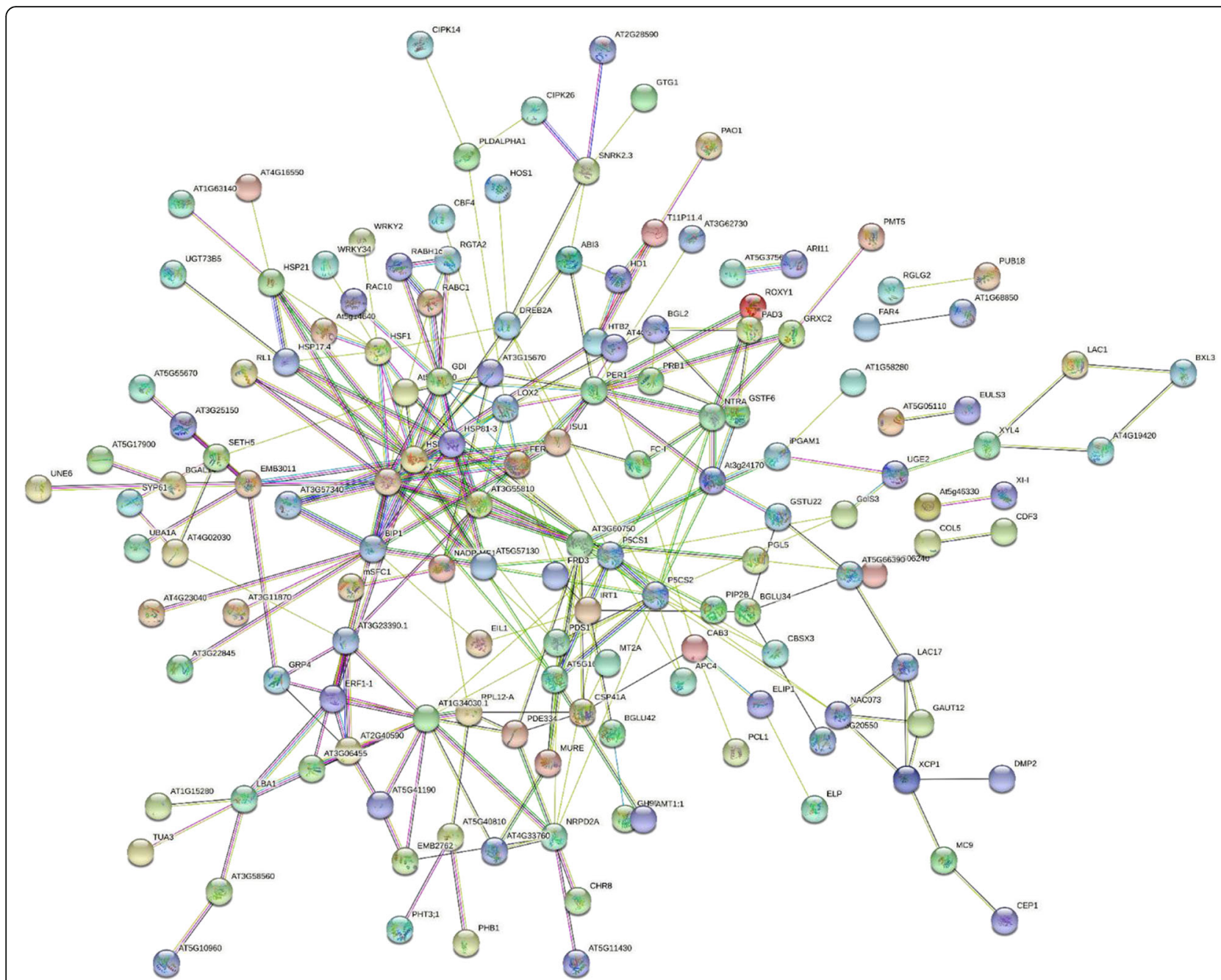

Fig. 1 Network of the genes with differential expression of $\geq 5$ and $-5 \geq$ at drought stress conditions plus their known neighbors in vegetative and reproductive organs of barley based on the microarray data using web based String software

1945 and 1854 putative binding sites were found for TFs with HD-ZIP and Trihelix domains in the 250 droughtresponsive genes.

\section{Cooperative modules analysis}

One module composed of two HD-ZIP transcription factors (Table 2) and was identified in $85 \%$ of the promoters (about 688 out of 212 DEGs). Also, six cooperative modules, i.e., DOF-C2C2 zinc finger domain, Trihelix domain, AT-hook, HD-ZIP, MYB domain, and Heterotrimeric were identified in the hub genes (Table 3). Besides, known modules (i.e., previously reported modules that are available in the database) were also searched in the promoter of the DEGs. According to the results, the binding site of an additional 261 modules in the DEGs (Supplementary Table 7) and 23 modules in the hub genes were identified (Supplementary Table 8). The most common module being identified among the droughtresponsive genes was the GATA_HNF1-01 module with the $\mathrm{C} 2 \mathrm{C} 2$ zinc finger domain transcription factor from the GATA binding factors family and the Hepatic Nuclear Factor from the homeodomain family which had 19 binding sites in 19 genes (Supplementary Table 7). Also, the most common module being identified in the promoter of hub genes was the GTBX_GTBX_01 Module, with six binding sites (Supplementary Table 8).

\section{Validation of the candidate genes}

The expression pattern of four promising candidate

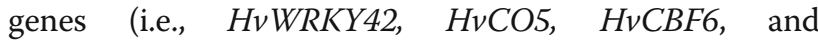
$H v D R F 1.3)$ which were found as hubs through the reconstruction of PPI networks and the hub analysis was evaluated by real-time PCR. Based on the results, the transcript levels of these genes were significantly 


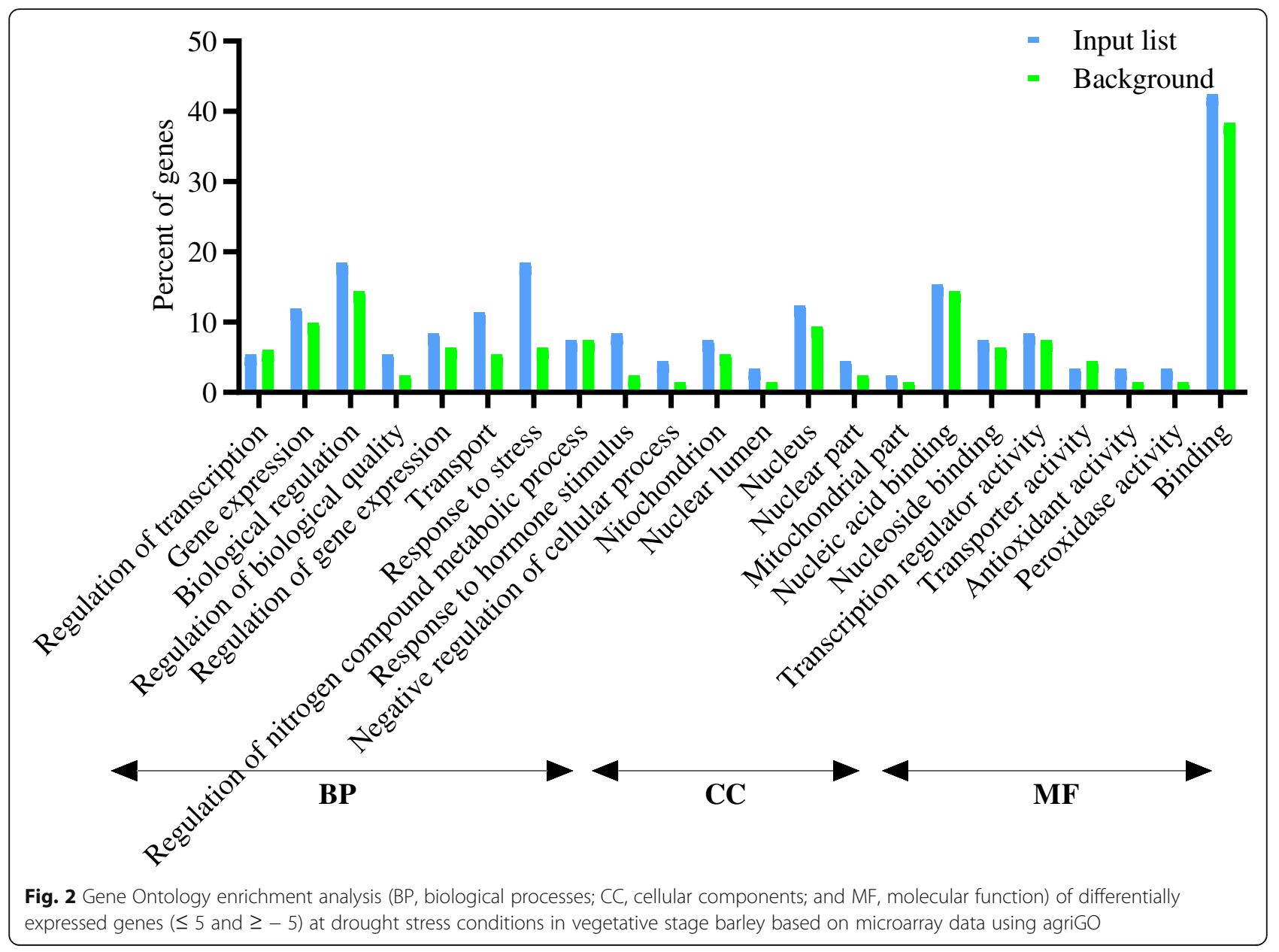

increased under drought conditions in the "Yousef" genotype, whereas the HvDRF1.3 gene was only induced in another genotype (Fig. 7). The results of real-time PCR showed that the expression pattern of the four genes was in good agreement with the results obtained by microarray analysis.

\section{Discussion}

In the present study, a total of 250 DEGs were obtained. Tommasini et al. [27] reported that in crown tissues of plants ( $5 \leq$ fold change $\leq-5$ ), 3330 genes were differentially expressed between the control group and drought stressed samples. A total of 144, 66, and 53 genes were differentially expressed $(P<0.0001, \mid \log 2$-fold $\mid$ value $\geq$ 2) between drought-stressed and control plants of Martin, HS41-1, and Moroc9-75, respectively, in at least one of the three time points [12]. Abebe et al. [13] studied the gene expression in the lemma, palea, awn, and seeds of barley under drought stress. The authors reported that there were only 16 upregulated and 15 downregulated genes common to all stressed organs $(q<$ 0.01 , $\mid \log 2$-fold $\mid$ value $\geq 2$ ). According to the literature review, the number of DEGs was mainly a function of the plant part used. Transcriptome data analysis in $H$. vulgare, $A$. thaliana $[27,35,36]$, and many other plants shows that tolerance or susceptibility to environmental stresses is mainly controlled at the level of transcription through the interaction between transcription factors in the regulatory networks. Transcription factors are master regulators that integrate, balance, and coordinate hormonal, developmental, and environmental signals in the plant systems [37]. According to the results of the present study (Supplementary Tables 3 and 4), HvWRKY42, HvEIL1, HvCBF6 (DREB1A), DRF1.3 (DREB2A), HvNAC6 (ATAF1), HvERF4, HvFUS3, $H \nu C O 5$ (HD1), and HvVP1 (ABI3) genes were among the hub genes of the PPI networks in the vegetative and reproductive stages. These genes were activated in response to drought stress (Table 4) and have been previously reported to be related to drought-stress response $[17,25,36,38-40]$.

The results of the present study confirmed that transcription factors play an important role in regulating the expression of genes that are responsible for responding 


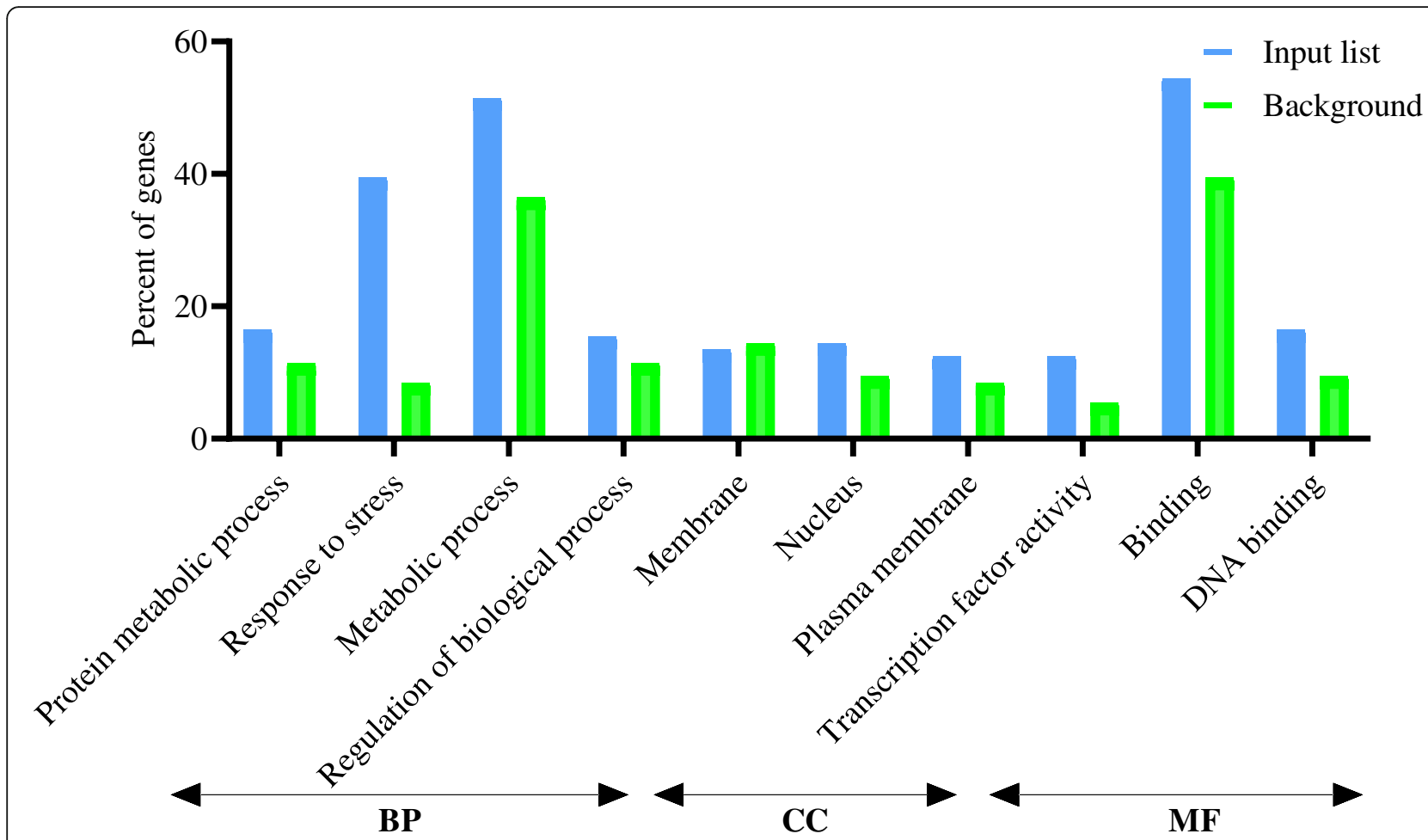

Fig. 3 Gene Ontology enrichment analysis (BP, biological processes; CC, cellular components; and MF, molecular function) of differentially expressed genes ( $\leq 5$ and $\geq-5$ ) at drought stress conditions in reproductive stage barley based on microarray data using agriGO

to drought stress. Transcription factors, as regulators of the target gene, are involved in many biological processes such as cell cycle growth, development, and response to environmental stresses. They cause the plant to adapt to adverse environmental conditions, including drought. The most important hub genes being identified in this study were transcription factors belonging to the AP2 family such as HvCBF6, HvDRF1.3, and VP1. These genes are involved in activating a complex drought tolerance network in barley [41]. The expression of the VP1 gene is stimulated by abscisic acid. Its protein product as a transcription factor regulates the expression of other genes during the middle and late stages of embryogenesis [42]. The WRKY transcription factor plays a very important role in regulating growth and response to biotic and abiotic stresses [43]. In tobacco, an increase in the expression of Tawrky gene through gene transfer caused enhancements in the plant's tolerance to drought and salinity stresses. The tolerance increased by regulating osmotic pressure and removing active oxygen species [44]. Also, a report suggested that the WRKY transcription factor increases tolerance against abiotic stresses by regulating stomatal opening and closing [45]. The four genes ( $H v W R K Y 42, H \nu C O 5, H \nu C B F 6$, and HvDRF1.3) were selected for evaluating at reproductive stage by real-time PCR based on three reasons: firstly, they were identified as potential hub genes which are transcription factors. Secondly, they belonged to different droughtresponsive gene families such as AP2 (HvCBF6, and HvDRF1.3), WRKY (HvWRKY42), and bZIP (HvCO5). Thirdly, drought stress affects all organs and developmental stages of the plants, but the reproductive stage is a critical stage as it determines the grain yield in barley $[12,13]$.

Interestingly, the gene expression analysis results of four potential candidate genes, i.e., $H v W R K Y 42, H \nu C B F 6$ (DREB1A), DRF1.3 (DREB2A), and HvCO5 (HD1) by real-time PCR in "Yousef" (the tolerant genotype) and Morocco (the susceptible genotype) indicated that all of the selected genes were upregulated in the tolerant genotype. Promoter analysis was conducted for further evaluation of regulatory factors in relation to drought stress in the plant. The AP2 family is one of the most important families of transcription factors and showed 827 ( $p$ value 3.58E-09) and 83 ( $p$ value 0.0011513 ) binding sites in the promoter of the drought-responsive genes. Also, it showed 47 ( $p$ value 4.10E-05) and 31 ( $p$ value 0.00062 ) binding sites in the promoter of the hub genes at the vegetative and reproductive stages, respectively. AP2 is one of the largest families of TFs being specific to the plant world [41]. The family has four subfamilies including AP2 (Apetala2), RAV, ERF, and DREB [46]. Among these, the subfamilies of DREB transcription factors often have a higher frequency in 


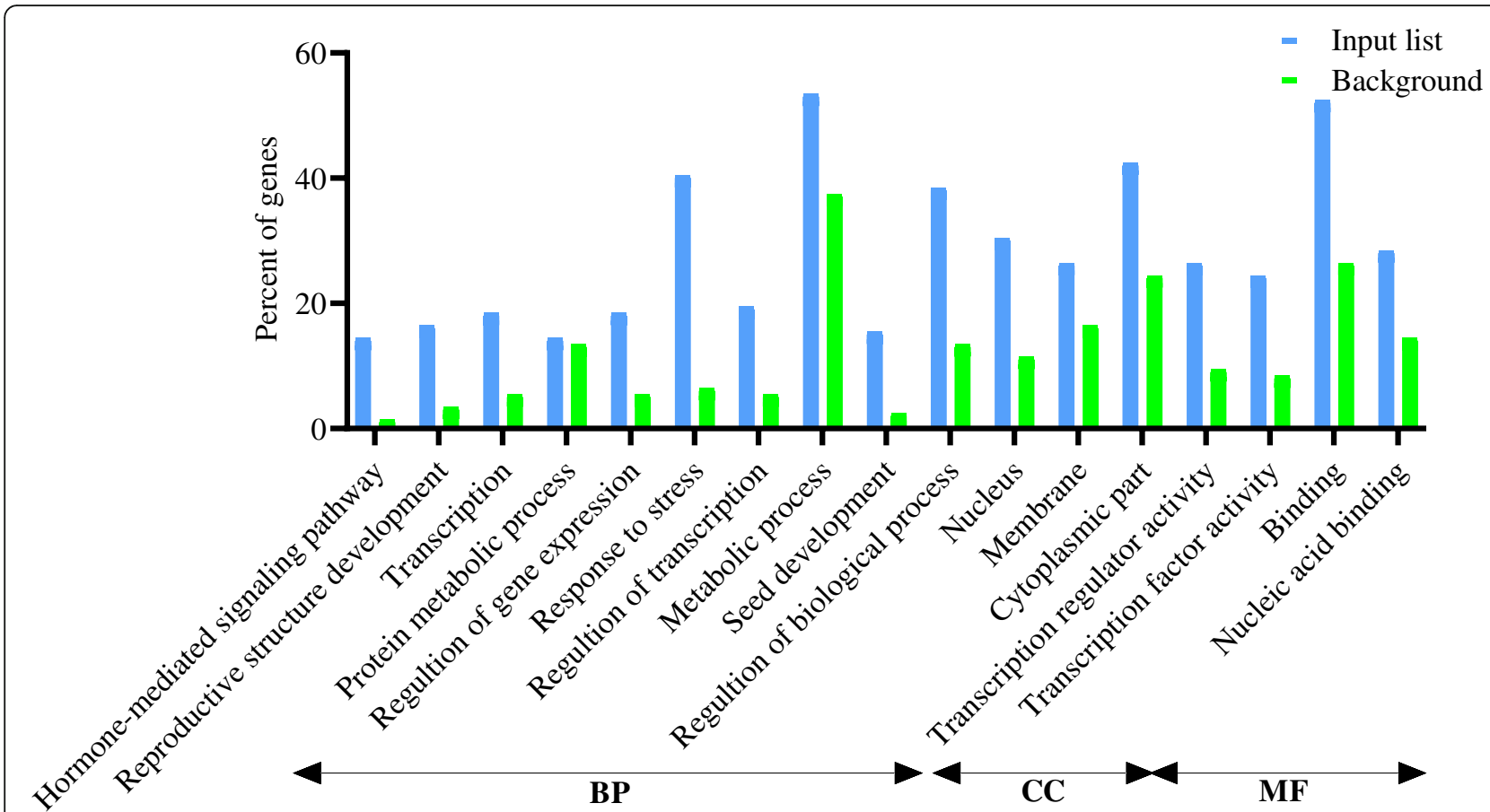

Fig. 4 Gene Ontology enrichment analysis (BP, biological processes; CC, cellular components; and MF, molecular function) of the determined hub genes at drought stress conditions in vegetative and reproductive stage barley based on microarray data using agriGO

response to different stresses such as drought, salinity, and rapid changes in temperature and disease, as compared to other subfamilies. Among the hub genes identified in the current research, $H v C B F 6$ (DREB1A), DRF1.3 (DREB2A), HvERF4, HvFUS3, and HvVP1 also belong to this family. The genes are known to activate the complex network of drought tolerance in the plant [41]. The overexpression of DREB/CBF genes in wheat and barley lead to an increase in plant tolerance to drought stress [47]. HvVP1 plays a crucial function during seed maturation and germination [48]. DRF1.3 and HvCBF6 genes specifically interact with the $H v V P 1$ to regulate the ABA response. HvEIL1 and HvFUS3 are key components of the seed development regulatory network and play an essential role in abscisic acid (ABA) responsive regulation of genes during the middle and final stages of embryogenesis [49]. During the maturation stage, grain reserves are accumulated and desiccation tolerance is achieved. This is actively controlled at the transcriptional level, and the AFL subfamily of B3 TFs plays a principal role through modulating hormone biosynthesis (mostly ABA and gibberellins) and other transcription factors of expression or their downstream activity via PPI [48]. NAC, bZIP, HD-ZIP, MYB, Zinc Finger, Trihelix, MADS, and WRKY families were also found to have binding sites in the promoter of a majority of the
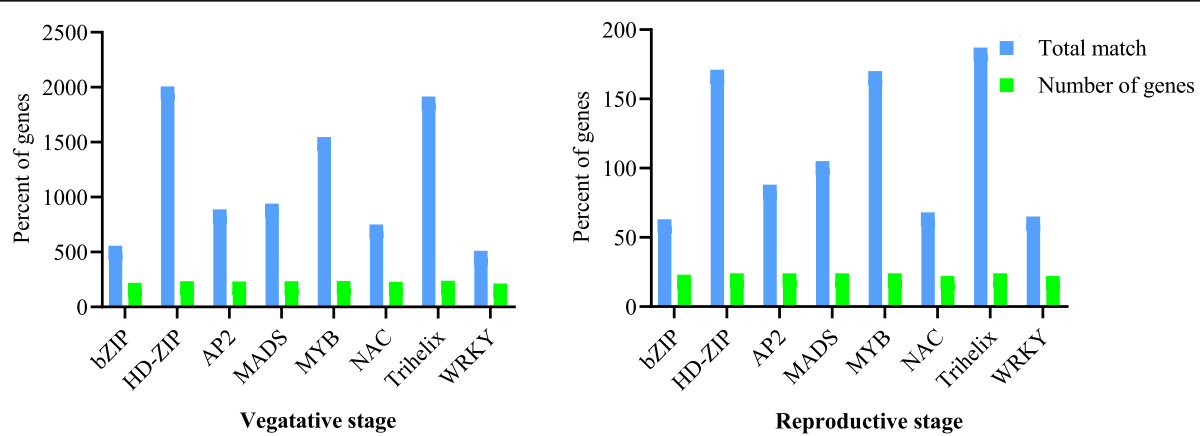

Fig. 5 Transcription factor families with binding sites in more than $85 \%(P$ value $<0.05)$ of the drought-responsive genes. Blue bar, the number of binding sites of these transcription factors predicted in the promoters of the DEGs; green bar, the number of genes having binding sites of these transcription factors in the promoters 


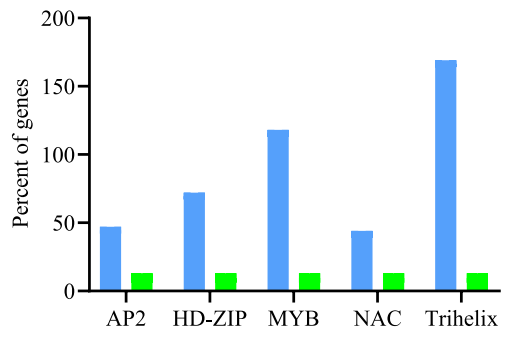

Vegatative stage

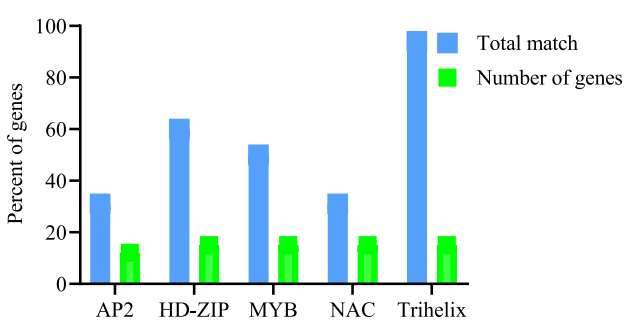

Reproductive stage

Fig. 6 Transcription factor families with binding sites in more than $85 \%$ ( $P$ value $<0.05)$ of the hub genes. Blue bar, the number of binding sites of these transcription factors predicted in the promoters of the hub genes; green bar, the number of hub genes having binding sites of these transcription factors in the promoters

drought-responsive genes. It can be justified by other reports that have shown associations between them and by the genes involved in drought tolerance [38, 50-56]. The MYB family is a diverse class of DNA-binding proteins that possess one conserved DNA-binding domain called MYB which has an important role in the regulation of gene transcription. Most MYB proteins are related to the regulation of plant responses to different stresses, hormone signaling, phenylpropanoid biosynthesis, and cell differentiation. Based on the results of the current study, the MYB family had 1484 ( $p$ value 0.00084 ) and 165 ( $p$ value 0.0149976 ) binding sites in the promoter of the drought-responsive genes, whereas 118 ( $p$ value $0.020252)$ and 50 ( $p$ value 0.015011$)$ binding sites in the promoter of the hub genes at the vegetative and reproductive stages, respectively.

Alexander et al. [23] studied the function of the barley transcription factor HvMYB1 and showed that gene expression is upregulated in wild-type barley roots and leaves under drought and osmotic stress. The authors also reported that transgenic barley plants that overexpress HvMYB1 were found to be more resistant to drought, showing enhanced relative water content, reduced water loss rate, and lower stomatal conductance, as compared to the control plants. Zhao et al. [57] reported that TaMYB31 genes have different tissue expression patterns, and based on RNA-seq analysis, it was revealed that TaMYB31 functions through the upregulation of wax biosynthesis genes and drought-responsive genes. The authors confirmed that the TaMYB31 acts as a positive regulator of drought resistance and, thus, justifies its potential application in the genetic modification of drought tolerance in crops.
NAC and bZIPs have important functions in the activation of ABA-dependent signaling pathways [53, 5860]. These families are upregulated under drought stress and some of them can increase drought tolerance due to the development of the root system [61]. Trihelix is a family of TFs and plays different roles in regulating the optical response, as well as the response to pathogens and abiotic stresses [62]. WRKY proteins are one of the largest families of transcription factors in plants and are mainly present in different biological processes such as the response to salinity and drought stress [40]. Our results showed that the HD-ZIP family of TFs had binding sites in 1945 ( $p$ value 4.41E-07) and 166 ( $p$ value $0.0083807)$ drought-responsive genes, along with $72(p$ value 0.00512$)$ and 60 ( $p$ value 0.014868$)$ hub genes at the vegetative and reproductive stages, respectively. Moreover, a cooperative module was composed of two HD-ZIP transcription factors. It was discovered in $85 \%$ of the drought-responsive promoters ( $P$ value $1.44434 \mathrm{E}$ $-15,688$ matches in 212 sequence) (Table 2). Previous studies have shown that the HD-ZIP family is a group of TFs in the plant world [63]. Janiak et al. [64] studied the barley root transcriptome under mild drought stress. The authors found 88 genes from 39 families involved in transcriptional regulation in roots upon mild drought. They were comprised of 13 gene TFs from the AP2 family represented by ERFs, DREB, or the B3 domaincontaining TFs, eight WRKYs, six NACs, five of the HDdomain, MYB, bHLH, and bZIP TFs. Members of this family contain a combination of a homeodomain and a leucine zipper in their structure. Although these two domains separately exist in other eukaryotes, their presence

Table 2 Cooperative module found in $85 \%$ of the drought responsive promoters

\begin{tabular}{lllllll}
\hline Module & Elements & Domain & Strand $\begin{array}{l}\text { Matrix } \\
\text { similarity }\end{array}$ & $\begin{array}{l}\text { Distance to next } \\
\text { element }\end{array}$ & Common to & $p$ value \\
\hline 1 & P\$AHBP & HD-ZIP & - & $0.85($ min. 0.85) & $9-18 \mathrm{bp}$ & $\begin{array}{l}688 \text { matches in 212 seq. (85\%), 532 non- } \\
\text { overlapping }\end{array}$ \\
\hline
\end{tabular}


Table 3 Cooperative modules found in the promoter of the hub genes

\begin{tabular}{|c|c|c|c|c|c|c|c|}
\hline Module & Elements & Domain & Strand & $\begin{array}{l}\text { Matrix } \\
\text { similarity }\end{array}$ & $\begin{array}{l}\text { Distance to next } \\
\text { element }\end{array}$ & Common to & $p$ value \\
\hline \multirow[t]{2}{*}{1} & P\$DOFF & DOF-C2C2 zinc finger domain & + & $\begin{array}{l}0.85(\min \\
0.96)\end{array}$ & $164-173 \mathrm{bp}$ & $\begin{array}{l}19 \text { matches in } 15 \text { seq. (88\%), } 18 \\
\text { non-overlapping }\end{array}$ & $\begin{array}{l}1.40355 \mathrm{e} \\
-06\end{array}$ \\
\hline & $P \$ G T B X$ & Trihelix domain & + & $\begin{array}{l}0.85(\min \\
0.86)\end{array}$ & & & \\
\hline \multirow[t]{2}{*}{2} & $P \$ H M G F$ & AT-hook & + & $\begin{array}{l}0.85(\min \\
0.88)\end{array}$ & $178-187 \mathrm{bp}$ & $\begin{array}{l}20 \text { matches in } 15 \text { seq. (88\%), } 17 \\
\text { non-overlapping }\end{array}$ & $\begin{array}{l}1.61627 e \\
-06\end{array}$ \\
\hline & P\$DOFF & DOF-C2C2 -zinc finger domain & + & $\begin{array}{l}0.85(\min \\
0.96)\end{array}$ & & & \\
\hline \multirow[t]{2}{*}{3} & $\mathrm{P} \$ \mathrm{AHBP}$ & $\mathrm{HD}-\mathrm{ZIP}$ & - & $\begin{array}{l}0.85(\min \\
0.86)\end{array}$ & $59-68 \mathrm{bp}$ & $\begin{array}{l}22 \text { matches in } 15 \text { seq. (88\%), } 19 \\
\text { non-overlapping }\end{array}$ & $\begin{array}{l}1.32362 \mathrm{e} \\
-05\end{array}$ \\
\hline & P\$SEF4 & $\begin{array}{l}\text { structure of DNA-binding domain } \\
\text { not specified }\end{array}$ & - & $\begin{array}{l}0.85(\min \\
0.91)\end{array}$ & & & \\
\hline \multirow[t]{2}{*}{4} & P\$AHBP & $\mathrm{HD}-\mathrm{ZIP}$ & + & $\begin{array}{l}0.85(\min \\
0.86)\end{array}$ & $173-182 \mathrm{bp}$ & $\begin{array}{l}34 \text { matches in } 16 \text { seq. (94\%), } 29 \\
\text { non-overlapping }\end{array}$ & $\begin{array}{l}2.11074 \mathrm{e} \\
-05\end{array}$ \\
\hline & P\$CAAT & heterotrimeric transcription factor & - & $\begin{array}{l}0.85(\min \\
0.86)\end{array}$ & & & \\
\hline \multirow[t]{2}{*}{5} & $\mathrm{P} \$ \mathrm{AHBP}$ & HD-ZIP & + & $\begin{array}{l}0.85(\min \\
0.86)\end{array}$ & $154-163$ bp & $\begin{array}{l}28 \text { matches in } 15 \text { seq. (88\%), } 24 \\
\text { non-overlapping }\end{array}$ & $\begin{array}{l}2.29321 \mathrm{e} \\
-05\end{array}$ \\
\hline & P\$GTBX & Trihelix domain & + & $\begin{array}{l}0.85(\min \\
0.85)\end{array}$ & & & \\
\hline \multirow[t]{2}{*}{6} & $P \$ H M G F$ & AT-hook & - & $\begin{array}{l}0.85 \text { (min. } \\
0.90\end{array}$ & $77-86 \mathrm{bp}$ & $\begin{array}{l}22 \text { matches in } 15 \text { seq. (88\%), } 22 \\
\text { non-overlapping }\end{array}$ & $\begin{array}{l}2.37645 \mathrm{e} \\
-05\end{array}$ \\
\hline & P\$MYBL & MYB domain & - & $\begin{array}{l}0.85(\min \\
0.85)\end{array}$ & & & \\
\hline
\end{tabular}
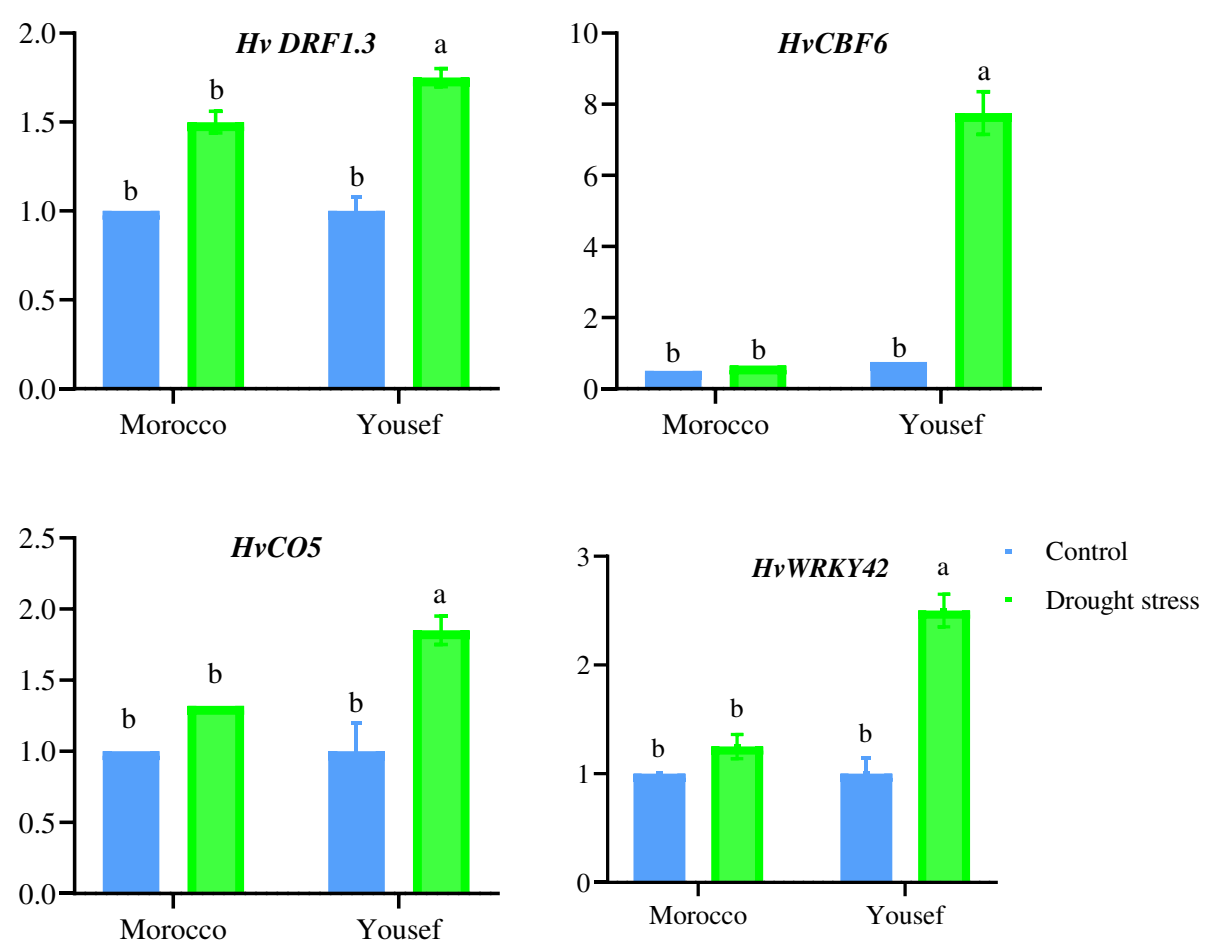

Fig. 7 The expression pattern of four promising candidate genes (HvDRFI.3, HvCBF6, HvCO5, and HvWRKY42) under drought conditions in $\mathrm{H}$. vulgare (Yousef, the tolerant, and Moroco, the sensitive cultivars) 
Table 4 The transcription factors which have been identified as hub genes

\begin{tabular}{llllll}
\hline Barley Uniprot ID & Barley gene name & Arabidopsis orthologs & Gene family & Algorithm & Tissue \\
\hline B2KJ82 & HvWRKY42 & WRKY70 & WRKY & MNC & Reproductive stage \\
Q3T5P4 & HvCBF6 & DREB1A & AP2 & MNC, Degree, Closeness & Reproductive stage \\
Q84JE9 & DRF1.3 & DREB2A & AP2 & MNC & Reproductive stage \\
E3VXB5 & HvEIL1 & EIL1 & EIN3 family & MNC, Degree, Closeness & Vegetative stage \\
F2DPJ4 & HVNAC6 & ATAF1 & NAC & MNC & Vegetative stage \\
F2D6Z2 & HvERF4 & ERF4 & AP2 & MNC & Vegetative stage \\
B1V8R3 & HVFUS3 & FUS3 & AP2 & Closeness & Vegetative stage \\
Q8LGM4 & HvCO5 & HD1 & Zinc-finger & Closeness/MNC & Vegetative stage/reproductive stage \\
Q8GV44 & HvVP1 & ABI3 & AP2 & Closeness & Vegetative stage \\
\hline
\end{tabular}

in the form of one protein is only observed in plants. The role of HD-ZIP proteins in hormonal signaling pathways or in response to different stresses such as drought has already been reported [65]. In the current research, the most common combinations of transcription factor binding sites (modules) were determined in the promoter of the drought-responsive genes and the hub genes (Tables 2 and 3). Studying different biological systems has shown that the highly delicate and specific, temporal-spatial regulation of gene expression is often achieved using cooperative models in which the expression of particular genes is regulated using the simultaneous effect of two or more transcription factors under certain circumstances [66]. Several structural and biochemical studies have shown various fundamental interactions among transcription factors and structural functions of promoter/enhancer in the coordinated regulation of eukaryotic genes [67]. These studies have proposed that cooperative regulation is the main mechanism in complex patterns of gene expression [68]. Thus, studying the modules instead of transcription factors alone can meet the complexity of the response to drought in barley.

\section{Conclusion}

The present study provided new insights into key genes associated with the plant response to drought stress in barley. This understanding occurred through the reconstruction of the related gene network and by the identification of the hub genes. Based on the results, a major proportion of the identified hub genes was involved in regulatory processes such as the regulation of transcriptions, e.g. HvVP1, HvNAC6, HvERF4, HvWRKY42, HvFUS3, HvCO5, HvCBF6, and DRF1.3, since these are from AP2, NAC, WRKY, and Homeobox transcription factor families. Moreover, a promoter analysis revealed that AP2, NAC, bZIP, WRKY, HD-ZIP and MYB are among the most common transcription factor families having a binding site in promoters of drought-responsive and hub genes. Therefore, biological processes such as regulation and gene expression play important roles in the tolerance of barley against drought stress. These insights can provide a new foundation for future research in improving plants to obtain new drought-tolerant genotypes.

\section{Supplementary Information}

The online version contains supplementary material available at https://doi. org/10.1186/s43141-020-00104-z.

\section{Additional file 1.}

Additional file 2.

Abbreviations

PPI: Protein-protein interaction; GEO: Gene Expression Omnibus; GO: Gene Ontology; DAA: Days after anthesis

\section{Acknowledgements}

We are grateful to ABRII for the supports.

Authors' contributions

ZSS conceived the research idea and designed the experiments. ZSS, AE, SMJ, and MS performed the experiments and analyzed the data. SMJ wrote the manuscript. The authors read and approved the final manuscript.

\section{Funding}

The present research was supported by ABRII with the grant no: 1-05-05519151-91001.

\section{Availability of data and materials}

All data generated or analyzed during this study are included in this published article.

\section{Ethics approval and consent to participate}

This article does not contain any studies with human participants or animals performed.

\section{Consent for publication \\ Not applicable}

\section{Competing interests}

The authors declare that they have no competing interests.

\section{Author details}

${ }^{1}$ Department of Biotechnology and Plant Breeding, Science and Research Branch, Islamic Azad University, Tehran, Iran. ${ }^{2}$ Department of Systems Biology, Agricultural Biotechnology Research Institute of Iran (ABRII), Agricultural Research, Education and Extension Organization (AREEO), Karaj, Iran. ${ }^{3}$ Gorgan University of Agricultural Sciences and Natural Resources, Gorgan, Iran. 
Received: 13 October 2020 Accepted: 14 December 2020 Published online: 06 January 2021

\section{References}

1. Ahmed IM, Dai H, Zheng W, Cao F, Zhang G, Sun D et al (2013) Genotypic differences in physiological characteristics in the tolerance to drought and salinity combined stress between Tibetan wild and cultivated barley. Plant Physiol Biochem 63:49-60. https://doi.org/10.1016/j.plaphy.2012.11.004

2. Hackenberg M, Gustafson P, Langridge P, Shi BJ (2015) Differential expression of microRNAs and other small RNAs in barley between water and drought conditions. Plant Biotechnol J 13:2-13 https://doi.org/10.1111/ pbi. 12220

3. Zhang X, Liu X, Wang W, Zhang T, Zeng X, Xu G et al (2018) Spatiotemporal variability of drought in the northern part of northeast China. Hydrol Process 32:1449-1460. https://doi.org/10.1002/hyp.11503

4. Alghabari F, Ihsan MZ (2018) Effects of drought stress on growth, grain filling duration, yield and quality attributes of barley (Hordeum vulgare L.). Bangladesh J Bot 47:421-428. https://doi.org/10.3329/bjb.v47i3.38679

5. Akbari KR, Husain R, Farajpour M, Mazzuca S, Mahna N (2020) Sodium chloride induced stress responses of antioxidative activities in leaves and roots of pistachio rootstock. Biomolecules 10. https://doi.org/10.3390/ biom10020189

6. Sheikh-Mohamadi MH, Etemadi N, Nikbakht A, Farajpour M, Arab M, Majidi MM (2017) Screening and selection of twenty iranian wheatgrass genotypes for tolerance to salinity stress during seed germination and seedling growth stage. HortScience 52:1125-1134. https://doi.org/10.21273/HORTSCl12103-17

7. Sheikh-Mohamadi MH, Etemadi N, Nikbakht A, Farajpour M, Arab M, Majidi MM (2018) Wheatgrass germination and seedling growth under osmotic stress. Agron J 110:572-585. https://doi.org/10.2134/agronj2017.06.0364

8. Arriagada O, Mora F, Quitral Y, Del Pozo A (2017) Identificação de QTL associados a características agronômicas, morfológicas e fisiológicas em cevada sob condições de sequeiro, usando marcadores SNP. Acta Sci Agron 39:321-329 https://doi.org/10.4025/actasciagron.v39i3.32612

9. Jin XF, Xiong AS, Peng RH, Liu JG, Gao F, Chen JM et al (2010) OsAREB1, an ABRE-binding protein responding to $A B A$ and glucose, has multiple functions in Arabidopsis. BMB Rep 43:34-39 https://doi.org/10.5483/BMBRep.2010.43.1.034

10. Ozturk ZN, Talamé V, Deyholos M, Michalowski CB, Galbraith DW, Gozukirmizi N et al (2002) Monitoring large-scale changes in transcript abundance in drought- and salt-stressed barley. Plant Mol Biol 48:551-573 https://doi.org/10.1023/A:1014875215580

11. Talamè V, Ozturk NZ, Bohnert HJ, Tuberosa R (2007) Barley transcript profiles under dehydration shock and drought stress treatments: a comparative analysis. J Exp Bot 58:229-240 https://doi.org/10.1093/jxb/erl163

12. Guo P, Baum M, Grando S, Ceccarelli S, Bai G, Li R et al (2009) Differentially expressed genes between drought-tolerant and drought-sensitive barley genotypes in response to drought stress during the reproductive stage. J Exp Bot 60:3531-3544 https://doi.org/10.1093/jxb/erp194

13. Abebe T, Melmaiee K, Berg V, Wise RP (2010) Drought response in the spikes of barley: Gene expression in the lemma, palea, awn, and seed. Funct Integr Genomics 10:191-205 https://doi.org/10.1007/s10142-009-0149-4

14. Worch S, Rajesh K, Harshavardhan VT, Pietsch C, Korzun V, Kuntze L et al (2011) Haplotyping, linkage mapping and expression analysis of barley genes regulated by terminal drought stress influencing seed quality. BMC Plant Biol 11:1 https://doi.org/10.1186/1471-2229-11-1

15. Hübner S, Korol AB, Schmid KJ (2015) RNA-Seq analysis identifies genes associated with differential reproductive success under drought-stress in accessions of wild barley Hordeum spontaneum. BMC Plant Biol 15:1-14 https://doi.org/10.1186/s12870-015-0528-z

16. Liang J, Chen X, Deng G, Pan Z, Zhang H, Li Q et al (2017) Dehydration induced transcriptomic responses in two Tibetan hulless barley (Hordeum vulgare var. nudum) accessions distinguished by drought tolerance. BMC Genomics 18:1-15 https://doi.org/10.1186/s12864-017-4152-1

17. Janiak A, Kwasniewski M, Sowa M, Gajek K, Żmuda K, Kościelniak J et al (2018) No time to waste: Transcriptome study reveals that drought tolerance in barley may be attributed to stressed-like expression patterns that exist before the occurrence of stress. Front Plant Sci 8 https://doi.org/10.3389/fpls.2017.02212

18. Yeger-lotem AE, Sattath S, Kashtan N, Itzkovitz S, Milo R, Pinter RY, et al. Protein Interaction Linked references are available on JSTOR for this article: network motifs in integrated cellular networks of transcription-regulation and protein-protein interaction 2016.
19. Fell DA, Wagner A (2000) The small world of metabolism. Nat Biotechnol 18: 1121-1122 https://doi.org/10.1038/81025

20. Guelzim N, Bottani S, Bourgine P, Képès F (2002) Topological and causal structure of the yeast transcriptional regulatory network. Nat Genet 31:6063 https://doi.org/10.1038/ng873

21. Freeling M, Subramaniam S (2009) Conserved noncoding sequences (CNSs) in higher plants. Curr Opin Plant Biol 12:126-132 https://doi.org/10.1016/j. pbi.2009.01.005

22. Sazegari S, Zinati Z, Tahmasebi A (2020) Dynamic transcriptomic analysis uncovers key genes and mechanisms involved in seed priming-induced tolerance to drought in barley. Gene Reports 21:100941

23. Alexander RD, Wendelboe-Nelson C, Morris PC (2019) The barley transcription factor HvMYB1 is a positive regulator of drought tolerance. Plant Physiol Biochem 142:246-253

24. Ju Y, Yue X, Min Z, Wang X, Fang Y, Zhang J (2020) VvNAC17, a novel stressresponsive grapevine (Vitis vinifera L.) NAC transcription factor, increases sensitivity to abscisic acid and enhances salinity, freezing, and drought tolerance in transgenic Arabidopsis. Plant Physiol Biochem 146:98-111

25. Yazdani B, Sanjari S, Asghari-Zakaria R, Ghanegolmohammadi F, Pourabed E, Shahbazi M et al (2020) Revision of the barley WRKY gene family phylogeny and expression analysis of the candidate genes in response to drought. Biol Plant 64:9-19

26. Cui $X, X u$ J, Asghar R, Condamine P, Svensson JT, Wanamaker S et al (2005) Detecting single-feature polymorphisms using oligonucleotide arrays and robustified projection pursuit. Bioinformatics 21:3852-3858

27. Tommasini L, Svensson JT, Rodriguez EM, Wahid A, Malatrasi M, Kato K et al (2008) Dehydrin gene expression provides an indicator of low temperature and drought stress: Transcriptome-based analysis of Barley (Hordeum vulgare L.). Funct Integr Genomics 8:387-405 https://doi.org/10.1007/ s10142-008-0081-z

28. Altschul SF, Madden TL, Schäffer AA, Zhang J, Zhang Z, Miller W et al (1997) Gapped BLAST and PSI-BLAST: a new generation of protein database search programs. Nucleic Acids Res 25:3389-3402 https://doi.org/10.1093/nar/25.17.3389

29. Szklarczyk D, Franceschini A, Wyder S, Forslund K, Heller D, Huerta-Cepas J et al (2015) STRING v10: Protein-protein interaction networks, integrated over the tree of life. Nucleic Acids Res 43:D447-D452 https:/doi.org/10.1093/nar/gku1003

30. Smoot ME, Ono K, Ruscheinski J, Wang PL, Ideker T (2011) Cytoscape 2.8: New features for data integration and network visualization. Bioinformatics 27:431-432 https://doi.org/10.1093/bioinformatics/btq675

31. Tian T, Liu Y, Yan H, You Q, Yi X, Du Z et al (2017) AgriGO v2.0: A GO analysis toolkit for the agricultural community, 2017 update. Nucleic Acids Res 45:W122-W129 https://doi.org/10.1093/nar/gkx382

32. Conesa A, Götz S, García-Gómez JM, Terol J, Talón M, Robles M (2005) Blast2GO: A universal tool for annotation, visualization and analysis in functional genomics research. Bioinformatics 21:3674-3676 https://doi.org/ 10.1093/bioinformatics/bti610

33. Schmittgen TD, Livak KJ (2008) Analyzing real-time PCR data by the comparative CT method. Nat Protoc 3:1101-1108 https://doi.org/10.1038/ nprot.2008.73

34. Pfaffl MW (2002) Relative expression software tool (REST(C)) for group-wise comparison and statistical analysis of relative expression results in real-time PCR. Nucleic Acids Res 30:36e https://doi.org/10.1093/nar/30.9.e36

35. Fowler S, Thomashow MF (2002) Arabidopsis transcrFowler, S., and Thomashow, M.F. (2002). Arabidopsis transcriptome profiling indicates that multiple regulatory pathways are activated during cold acclimation in addition to the CBF cold response pathway. Plant Cell 14: 1675-90. iptome pr. Plant Cell 14:1675-1690 https://doi.org/10.1105/tpc.003483. Toward

36. Umezawa T, Fujita M, Fujita Y, Yamaguchi-Shinozaki K, Shinozaki K (2006) Engineering drought tolerance in plants: discovering and tailoring genes to unlock the future. Curr Opin Biotechnol 17:113-122 https://doi.org/10.1016/ j.copbio.2006.02.002

37. Sah SK, Reddy KR, Li J (2016) Abscisic acid and abiotic stress tolerance in crop plants. Front Plant Sci 7:1-26 https://doi.org/10.3389/fpls.2016.00571

38. Gürel F, Öztürk ZN, Uçarlı C, Rosellini D (2016) Barley Genes as Tools to Confer Abiotic Stress Tolerance in Crops. Front Plant Sci 7 https://doi.org/10. 3389/fpls.2016.01137

39. Joshi R, Wani SH, Singh B, Bohra A, Dar ZA, Lone AA et al (2016) Transcription factors and plants response to drought stress: current understanding and future directions. Front Plant Sci 7:1-15 https://doi.org/ 10.3389/fpls.2016.01029 
40. Wehner G, Balko C, Humbeck K, Zyprian E, Ordon F (2016) Expression profiling of genes involved in drought stress and leaf senescence in juvenile barley. BMC Plant Biol 16:1-12 https://doi.org/10.1186/s12870-015-0701-4

41. Mizoi J, Shinozaki K, Yamaguchi-Shinozaki K (1819) AP2/ERF family transcription factors in plant abiotic stress responses. Biochim Biophys Acta - Gene Regul Mech 2012:86-96 https://doi.org/10.1016/j.bbagrm.2011.08.004

42. Hu H, Dai M, Yao J, Xiao B, Li X, Zhang Q et al (2006) Overexpressing a NAM, ATAF, and CUC (NAC) transcription factor enhances drought resistance and salt tolerance in rice. Proc Natl Acad Sci 103:12987-12992

43. Mingyu Z, Zhengbin Z, Shouyi C, Jinsong Z, Hongbo S (2012) WRKY transcription factor superfamily: structure, origin and functions. Afr J Biotechnol 11:8051-8059

44. Wang N-N, Xu S-W, Sun Y-L, Liu D, Zhou L, Li Y et al (2019) The cotton WRKY transcription factor (GhWRKY33) reduces transgenic Arabidopsis resistance to drought stress. Sci Rep 9:1-13

45. Li S, Fu Q, Huang W, Yu D (2009) Functional analysis of an Arabidopsis transcription factor WRKY25 in heat stress. Plant Cell Rep 28:683-693

46. Cao S, Wang Y, Li X, Gao F, Feng J, Zhou Y (2020) Characterization of the AP2/ERF transcription factor family and expression profiling of DREB subfamily under cold and osmotic stresses in Ammopiptanthus nanus. Plants 9:1-20 https://doi.org/10.3390/plants9040455

47. Morran S, Eini O, Pyvovarenko T, Parent B, Singh R, Ismagul A et al (2011) Improvement of stress tolerance of wheat and barley by modulation of expression of DREB/CBF factors. Plant Biotechnol J 9:230-249 https://doi. org/10.1111/j.1467-7652.2010.00547.x

48. Abraham Z, Iglesias-Fernández R, Martínez M, Rubio-Somoza I, Díaz I, Carbonero P et al (2016) A developmental switch of gene expression in the barley seed mediated by HvVP1 (Viviparous-1) and HvGAMYB interactions. Plant Physiol 170:2146-2158 https://doi.org/10.1104/pp.16.00092

49. Mönke G, Seifert M, Keilwagen J, Mohr M, Grosse I, Hähnel U et al (2012) Toward the identification and regulation of the Arabidopsis thaliana ABI3 regulon. Nucleic Acids Res 40:8240-8254 https://doi.org/10.1093/nar/gks594

50. He GH, Xu JY, Wang YX, Liu JM, Li PS, Chen M et al (2016) Droughtresponsive WRKY transcription factor genes TaWRKY1 and TaWRKY33 from wheat confer drought and/or heat resistance in Arabidopsis. BMC Plant Biol 16:1-16 https://doi.org/10.1186/s12870-016-0806-4

51. Harris JC, Hrmova M, Lopato S, Langridge P (2011) Modulation of plant growth by HD-Zip class I and II transcription factors in response to environmental stimuli. New Phytol 190:823-837 https://doi.org/10.1111/j. 1469-8137.2011.03733.x

52. Zhang S, Haider I, Kohlen W, Jiang L, Bouwmeester H, Meijer AH et al (2012) Function of the HD-Zip I gene Oshox22 in ABA-mediated drought and salt tolerances in rice. Plant Mol Biol 80:571-585 https://doi.org/10.1007/s11103012-9967-1

53. Puranik S, Sahu PP, Srivastava PS, Prasad M (2012) NAC proteins: regulation and role in stress tolerance. Trends Plant Sci 17:369-381 https://doi.org/10. 1016/j.tplants.2012.02.004

54. Christiansen MW, Holm PB, Gregersen PL (2011) Characterization of barley (Hordeum vulgare L.) NAC transcription factors suggests conserved functions compared to both monocots and dicots. BMC Res Notes 4:1-13 https://doi.org/10.1186/1756-0500-4-302

55. Huang J, Sun SJ, Xu DQ, Yang X, Bao YM, Wang ZF et al (2009) Increased tolerance of rice to cold, drought and oxidative stresses mediated by the overexpression of a gene that encodes the zinc finger protein ZFP245. Biochem Biophys Res Commun 389:556-561 https://doi.org/10.1016/j.bbrc.2009.09.032

56. Sanjari S, Shirzadian-Khorramabad R, Shobbar ZS, Shahbazi M (2019) Systematic analysis of NAC transcription factors' gene family and identification of postflowering drought stress responsive members in sorghum. Plant Cell Rep 38: 361-376 https://doi.org/10.1007/s00299-019-02371-8

57. Zhao Y, Cheng X, Liu X, Wu H, Bi H, Xu H (2018) The wheat MYB transcription factor TaMYB31 is involved in drought stress responses in Arabidopsis. Front Plant Sci 9:1426

58. Nakashima K, Ito Y, Yamaguchi-Shinozaki K (2009) Transcriptional regulatory networks in response to abiotic stresses in Arabidopsis and grasses. Plant Physiol 149:88-95 https://doi.org/10.1104/pp.108.129791

59. Yang A, Dai X, Zhang WH (2012) A R2R3-type MYB gene, OsMYB2, is involved in salt, cold, and dehydration tolerance in rice. J Exp Bot 63:25412556 https://doi.org/10.1093/jxb/err431

60. Liu C, Mao B, Ou S, Wang W, Liu L, Wu Y et al (2014) OsbZIP71, a bZIP transcription factor, confers salinity and drought tolerance in rice. Plant Mol Biol 84:19-36 https://doi.org/10.1007/s11103-013-0115-3
61. Jeong JS, Kim YS, Baek KH, Jung H, Ha SH, Choi Y Do, et al. (2010) Rootspecific expression of OsNAC10 improves drought tolerance and grain yield in rice under field drought conditions. Plant Physiol 153:185-197 https://doi. org/10.1104/pp.110.154773

62. Kaplan-Levy RN, Brewer PB, Quon T, Smyth DR (2012) The trihelix family of transcription factors - light, stress and development. Trends Plant Sci 17: 163-171 https://doi.org/10.1016/j.tplants.2011.12.002

63. Perotti MF, Ribone PA, Chan RL (2017) Plant transcription factors from the homeodomain-leucine zipper family I. Role in development and stress responses. IUBMB Life 69:280-289 https://doi.org/10.1002/iub.1619

64. Janiak A, Kwasniewski M, Sowa M, Kuczyńska A, Mikołajczak K, Ogrodowicz P et al (2019) Insights into barley root transcriptome under mild drought stress with an emphasis on gene expression regulatory mechanisms. Int J Mol Sci 20:6139

65. Ariel FD, Manavella PA, Dezar CA, Chan RL (2007) The true story of the HD-Zip family. Trends Plant Sci 12:419-426 https://doi.org/10.1016/j.tplants.2007.08.003

66. Balaji S, Babu MM, lyer LM, Luscombe NM, Aravind L (2006) Comprehensive analysis of combinatorial regulation using the transcriptional regulatory network of yeast. J Mol Biol 360:213-227 https://doi.org/10.1016/j.jmb.2006.04.029

67. Klinkenberg LG, Mennella TA, Luetkenhaus K, Zitomer RS (2005) Combinatorial repression of the hypoxic genes of Saccharomyces cerevisiae by DNA binding proteins Rox1 and Mot3. Society 4:649-660 https://doi.org/ 10.1128/EC.4.4.649

68. Banerjee N, Zhang MQ (2003) Identifying cooperatively among transcription factors controlling the cell cycle in yeast. Nucleic Acids Res 31:7024-7031 https://doi.org/10.1093/nar/gkg894

\section{Publisher's Note}

Springer Nature remains neutral with regard to jurisdictional claims in published maps and institutional affiliations.

\section{Submit your manuscript to a SpringerOpen ${ }^{\circ}$ journal and benefit from:}

- Convenient online submission

- Rigorous peer review

- Open access: articles freely available online

- High visibility within the field

- Retaining the copyright to your article

Submit your next manuscript at $\boldsymbol{\nabla}$ springeropen.com 